\title{
Utilizing computer assisted laboratory procedures in pharmacology remotely: teaching \& learning at times of uncertainty background Computer Assisted Learning (CAL)
}

\author{
Htet, H., Agarwal, R., Ayob, A., lezhitsa, I.N., Yew, O.T.H., Ismail, H.J.N.M. \\ International Medical University, Malaysia
}

\section{Background:}

Utilizing Computer Assisted Laboratory Procedures in Pharmacology Remotely: Teaching \& Learning at Times of Uncertainty Background Computer assisted learning (CAL) is an important tool for the learning process through use of software to understand the concepts and techniques (1). With a trend towards abolishing use of animals for undergraduate teaching, CAL sessions are cornerstone for delivering essential conceptual knowledge in disciplines such as pharmacology. In School of Medicine, International Medical University, one CAL session has been incorporated for year 1 medical students. At the end of the session, we expect the students to be able to explain the concepts of drug agonism and antagonism, perform the experiment using computer software and apply these concepts to understand the clinical problems.

\section{Method:}

During the unprecedented time of COVID 19 and movement control order imposed in Malaysia, the CAL session was conducted online through video demonstration and interactive discussion. Screen recording of the entire practical was done by department members and converted to video format. After the session, students' feedbacks were collected through google form. Out of 200 students, 79 responses were obtained. Data analysis was done using Microsoft excel.

\section{Results:}

Among all, $89.8 \%(n=71)$ of respondents agree or strongly agree that the CAL practical session met the stated objective (Q1); $92.4 \%(n=73)$ of respondents agree or strongly agreed that the CAL session was useful for them to understand general pharmacology better (Q2); 81\% $(n=64)$ of respondents agree or strongly agree that they were more confident about answering general pharmacology question after the CAL session (Q3); $89.8 \%(n=71)$ of respondents agree or strongly agree that the organization was good (Q4) and $92.4 \%(n=73)$ of the respondents agree or strongly agree that the facilitators were helpful to achieve the stated lesson outcomes (Q5) (Figure 1). Regarding the overall rating, $44.3 \%(n=35)$ responded as very good, $31.7 \%(n=25)$ responded as good, $20.3 \%(n=16)$ responded as excellent and $3.8 \%(n=3)$ responded as fair (Figure 2). Overall, the open feedbacks by majority of the students were positive.

\section{Conclusion:}

Generally, students were satisfied with the online version of CAL session. They believed that the objectives were achieved as well as organization, conduct by the facilitators, and overall usefulness was appropriate. Qualitative open feedbacks are positive with some suggestions. Overall, conducting a CAL session through online platform during an unprecedented time was challenging and the new approach by the department was positively perceived by students.

Key words: Teaching Learning, Pharmacology, COVID-19 